Cahiers de philosophie de l'université de

\title{
Alexeï Lossev lecteur de Platon : enquête sur l'herméneutique platonicienne
}

\section{Olga Kobenko}

\section{(2) OpenEdition}

1 Journals

Édition électronique

URL : https://journals.openedition.org/cpuc/1002

DOI : $10.4000 /$ cpuc. 1002

ISSN : 2677-6529

Éditeur

Presses universitaires de Caen

\section{Édition imprimée}

Date de publication : 31 octobre 2011

Pagination : 151-172

ISBN : 978-2-84133-385-1

ISSN : 1282-6545

\section{Référence électronique}

Olga Kobenko, « Alexeï Lossev lecteur de Platon : enquête sur l'herméneutique platonicienne », Cahiers de philosophie de l'université de Caen [En ligne], 48 | 2011, mis en ligne le 01 septembre 2020, consulté le 02 février 2023. URL : http://journals.openedition.org/cpuc/1002 ; DOI : https://doi.org/10.4000/ cpuc. 1002

\section{(c) (7) (8)}

Creative Commons - Attribution - Pas d'Utilisation Commerciale 4.0 International - CC BY-NC 4.0 https://creativecommons.org/licenses/by-nc/4.0/ 


\section{Alexeï Lossev lecteur de Platon : enquête sur l'herméneutique platonicienne}

$\mathrm{E}^{\mathrm{N}}$ Nussie, au tout début de l'époque bolchevique, le philosophe et philologue Alexeï Lossev (1893-1988) s'est consacré à la tâche ardue de la compréhension de l'œuvre de Platon à partir de prémisses proprement philosophiques qui l'ont placé lui-même dans le sillage de la tradition platonicienne. Pour un penseur moderne, philosopher dans l'esprit de Platon semble être quelque chose de fort différent de la tâche de l'interpréter en tant qu'historien. Lossev voyait pourtant les choses autrement: selon lui, comme avant lui, selon Plotin ou Proclus, afin de comprendre Platon, il faut être soi-même platonicien. Quel contresens, quel abus des «axiomes" de l'herméneutique des textes classiques, pourrait-on dire aujourd'hui ! Mais en fin de compte avait-il tellement tort? On se propose ici d'analyser les prémisses méthodologiques sur lesquelles repose la lecture lossevienne de Platon en s'interrogeant sur les questions herméneutiques qu'elle fait surgir, si l'on entend par herméneutique une « réflexion sur les opérations de compréhension impliquées dans l'interprétation des textes » ${ }^{1}$.

Rien de plus singulier et inattendu au premier regard qu'une lecture de Platon faite par un auteur russe dans les années 1920. D’une importance mineure, toute nationale, semble-t-il, au premier abord, la portée de cette question ne dépendrait que du rôle que cet auteur a joué dans son époque et dans son pays. Ou bien, dans le meilleur des cas de figure, elle pourrait susciter un intérêt relativement général dans le cadre d'une étude de la réception de l'œuvre platonicienne à travers diverses cultures et différentes époques. Le Platon de Lossev se présenterait alors comme une curiosité historique, un anachronisme, quelque chose de dépassé, surtout si on le mesure à l'aune du progrès de la science d'aujourd'hui. Cependant les choses

1. P. Ricœur, Du texte à l'action. Essais d'herméneutique. II, Paris, Seuil, 1986, p. 75. 
semblent se présenter autrement. La lecture de Platon par Lossev mérite un intérêt propre qu'elle peut revendiquer pour elle-même. Il en est ainsi principalement en raison du fait que cette lecture est intentionnellement philosophique. «Je conçois mon travail comme n'étant absolument pas philologique. C'est un travail historico-philosophique $»^{2}$. Il en est ainsi dans la mesure où la méthode adoptée par Lossev est en même temps en grande partie hérétique à l'égard de son cadre disciplinaire: un puriste dans le domaine de l'histoire de la philosophie serait sans doute indigné par la facilité avec laquelle Lossev saute de Plotin à la théorie de la relativité d'Einstein ${ }^{3}$, ou encore pourrait être surpris de découvrir dans un ouvrage presque entièrement consacré à Platon la question du filioque qui témoigne aux yeux de Lossev d'une faille logique inhérente au catholicisme ${ }^{4}$.

L'aspect le plus intéressant et le plus troublant de l'interprétation de Platon par Lossev consiste en ce que l'auteur pose des problèmes philosophiques en termes exégétiques, de sorte qu'on peut parler de la renaissance du paradigme "exégétique ${ }^{5}$ dans la philosophie russe au début de l'époque soviétique. En quoi Lossev, historien de la philosophie grecque et singulièrement de Platon, s'apparente-t-il aux commentateurs de l'Antiquité tardive? Pourquoi a-t-il adopté ce mode «exégétique » de pensée qui parait, à certains du moins, désuet et étrange aujourd'hui?

Alexeï Lossev a donc mis en œuvre, au début du XXe siècle, le projet ambitieux de reprendre à son compte l'ensemble de la philosophie grecque, depuis Thalès et Anaximandre jusqu'à Proclus et Olympiodore, tout en jetant en même temps les bases de son propre système philosophique. Le fruit de ce travail mené avec la fougue et la véhémence du jeune âge ${ }^{6}$ a pris la forme d'une série de huit monographies publiées entre 1927 et $1930^{7}$.

2. A. Lossev, Ocherki antichnogo simvolizma i mifologii [Essais sur le symbolisme et la mythologie antiques], Moskva, Mysl', 1993, p. 139.

3. Voir A. Lossev, Antichnii kosmos i sovremennaia nauka [Cosmos antique et science contemporaine], in Bytie, imia, kosmos [Être, nom, cosmos], Moskva, Mysl', 1993, p. 62-612.

4. A. Lossev, Ocherki..., p. 875-877.

5. Voir au sujet de la philosophie «exégétique» l'article de P. Hadot, «Philosophie, exégèse et contresens», in Études de philosophie ancienne, Paris, Les Belles Lettres, 1998, p. 3-11.

6. Dans l'introduction aux Essais sur le symbolisme et la mythologie antiques publiés en 1930 Lossev affirme les avoir écrits dix ans avant la date de publication, c'est-à-dire, si l'on croit cet aveu, qu'il faut considérer qu'il avait 27 ans lors de la rédaction de cet ouvrage fondamental autant pour sa lecture de Platon que pour son interprétation de la philosophie grecque en général.

7. Dans l'ordre chronologique ce corpus se présente comme suit: 1927: Antichnii kosmos i sovremennaia nauka [Le cosmos antique et la science contemporaine]; Filosofia imeni [Philosophie du nom] ; Dialektika hudojestvennoi formy [Dialectique de la forme artistique]; Muzyka kak predmet logiki [Musique comme objet de la logique]; 1928: Dialektika chisla u 
Ce corpus contient en apparence deux blocs thématiques: d'un côté une partie historico-philosophique, et d'un autre une partie analytique et thématique. La première partie, composée de quatre monographies ${ }^{8}$ traite de la philosophie grecque, plus spécifiquement du néoplatonisme, tandis que l'autre ${ }^{9}$ est consacrée à l'analyse de diverses formes symboliques, telles que le langage, le mythe, l'art, etc. Cette division est également confirmée par un historien de la philosophie russe, Serge Khorujii, qui formule les objectifs du corpus lossevien en ces termes:

Les problèmes multiples que se pose le cycle lossevien peuvent se ramener, en condensant fortement, à deux objectifs principaux: la reconstruction de la pensée antique, et la constitution d'un système original de philosophie dialectique et phénoménologique, fondé sur des conceptions nouvelles du nom, du symbole et du mythe ${ }^{10}$.

Or, cette dichotomie thématique ne peut en aucun cas être maintenue en tant que division radicale: ses deux volets se recoupent. L'élément historico-philosophique n'est pas complètement séparable de l'élément "personnel et philosophique» et, mutatis mutandis, ce dernier n'est pas davantage complètement autonome par rapport au premier. On perçoit dès une première lecture quelque peu approfondie que, d'une part, les ouvrages historico-philosophiques de Lossev reprennent d'un bout à l'autre la problématique des ouvrages théorétiques, et que, d'autre part, l'expression de la philosophie personnelle de Lossev tend à devenir une paraphrase des auteurs grecs, néoplatoniciens plus particulièrement. En outre, Lossev soumet les textes grecs à une exégèse tellement vertigineuse qu'on ne reconnaît pas toujours ni tout à fait les auteurs dans leur littéralité derrière les artifices de l'interprétation.

Prenons, par exemple, la conclusion troublante des plus de cinq cents pages consacrées à l'analyse de la théorie platonicienne des Idées, menée sur la base d'une étude détaillée du texte de l'ensemble de dialogues: «L'idée

Plotina [La dialectique du nombre chez Plotin] ; 1929: Kritika platonizma u Aristotela [La critique du platonisme chez Aristote] ; 1930: Ocherki antichnogo simvolizma i mifologii [Essais sur le symbolisme et la mythologie antiques]; Dialektika mifa [La dialectique du mythe].

8. Le cosmos antique et science contemporaine, La dialectique du nombre chez Plotin, La critique du platonisme chez Aristote et Essais sur le symbolisme et la mythologie antiques.

9. La musique comme objet de la logique, Philosophie du nom, Dialectique de la forme artistique et La dialectique du mythe.

10. S. S. Khorouji', "Ariergardnii boï: misl' i mif Aleksieia Losseva », Voprosy filosofii, no 10, 1992, p. 76 : «Многообразные задачи, которые ставит перед собою лосевский цикл, можно, обобщая, свести к двум крупнейшим: реконструкция античного мышления и миросозерцания и создание оригинальной системы диалектико-феноменологической философии, имеющей в своей основе новые концепции имени, символа и мифа ». 
c'est l'Eidos, l'Intellect, engendré par l'autoaffirmation se niant elle-même (à l'aide du nombre) de l'Un qui transcende l'être; il engendre à son tour, de la même façon, l'Âme, et à travers elle, le Cosmos; il devient ainsi le Mythe sophiano-symbolique ${ }^{11}$. Lossev précise heureusement, tout de suite après, que Platon «ou bien n'utilise pas du tout cette terminologie, ou bien l'emploie dans un sens spécifique $»^{12}$. Mais s'agit-il là de Platon ou de Plotin? Ou serait-ce Proclus?

On peut également être surpris par les «quatre hypostases» du résumé suivant: "Le platonisme est la doctrine de trois, ou si l'on veut, quatre hypostases qui déploient l'être dans sa totalité: l'Un, l'Intellect (l'Eidos, l'Idée), l'Âme et le Cosmos» ${ }^{13}$.

Inversement, lorsque Lossev parle en son propre nom, dans les ouvrages qu'il consacre aux questions générales et théoriques, comme celle du mythe, de l'art ou du langage, ces thèmes s'inspirent largement des sources grecques. Par exemple, dans Dialectique de la forme artistique, Lossev commence par le constat suivant: «Le commencement de la dialectique est le nonpensable, l'hyper-pensable ${ }^{14}$, l'unicité absolue, et non pas tel ou tel objet, ni en général une chose quelconque, mais bien la puissance de toutes les choses et de toutes les catégories ${ }^{15}$. S'agit-il là d'esthétique ou bien du Parménide de Platon lu avec les lunettes de Plotin? On pourrait multiplier les exemples indéfiniment. Ce n'est pas pour autant notre but. L'important est de constater que les deux objectifs que M. Horujii entrevoit dans les recherches losseviennes des années 1920, la «reconstruction de la pensée antique» et la «constitution d'un système original de philosophie dialectique et phénoménologique» ne font en réalité qu'un. On a l'impression d'être face à l'œuvre d'un philosophe-exégète qui fond, en un seul mouvement de lecture, l'étude philologique, l'interprétation philosophique et la pensée personnelle.

11. A. Lossev, Ocherki..., p. 621: «Идея есть порожденный путем самотрицающегося (при помощи числа) самоутверждения сверх-сущего Единого Эйдос, Ум, который в свою очередь порождая из себя тем же путем Душу, а через нее и Космос, становится софийно-символическим Мифом».

12. Ibid.: «Платон сам или совсем не употребляет эту терминологию, или употребляет ее в специфическом значении ».

13. Ibid., p. 620: «Платонизм есть учение о трех, или если хотите, о четырех ипостасях, диалектически развертывающих бытие во всей его целости, о Едином, Уме (Эйдосе, Идее), Душе и Космосе».

14. La notion d' «hyper-pensable», ou d' "hyper-pensée» (en grec hupernoèsis) se trouve dans le traité de Plotin, Sur la liberté et la volonté de l'Un, VI, 8 [39], 16, 32, in Ennéades.

15. A. Lossev, Forma, stil, virajenie, Moskva, Mysl', 1995, p. 11: «Начало диалектики немыслимость, вышемыслимость, абсолютная единичность, которая не есть ни то, ни это, вообще никакая отдельная вещь, но - потенция всех вещей и категорий ». 
Mais peut-être ne s'agit-il là que des fautes d'un profane, d'un amateur qui n'avait pas su maîtriser les «axiomes» de l'herméneutique des textes classiques? Ou bien faut-il expliquer ce phénomène par le contexte spécifiquement russe, en soulignant en particulier le retard de plusieurs siècles mis par la Russie à intégrer la communauté scientifique européenne? En ce qui concerne le manque de culture philologique, ou tout simplement universitaire, chez Lossev, il faut, semble-t-il, écarter d'emblée un tel soupçon. L'essor des sciences humaines en Russie au tournant du siècle, son intégration croissante et très avancée dans la communauté scientifique européenne ne nous permettent pas de penser qu'il s'agit là seulement d'une situation propre aux savants russes.

Frances Nethercott écrit: "Today most historians of Russia would agree that the striking progress in Russian humanities scholarship in the closing decades of the nineteenth century was, to a great extent, due to more sustained interaction and exchange with the European scientific community as a whole $»^{16}$. Les lettres classiques furent particulièrement privilégiées à l'époque où Lossev fit ses études, et cela aussi bien au niveau de l'enseignement préuniversitaire, assuré par les gymnasium (avec quarante pour cent des cours consacrés à l'apprentissage de la grammaire grecque et latine ${ }^{17}$ ), qu'à l'Université où, depuis la contre-réforme de 1884 , l'enseignement de la philosophie, de la littérature et de l'histoire était subordonné à la philologie classique. "A direct - and at first glance surprising - consequence of the Counter Reform on university instruction in the humanities was the subordination of philosophy, along with literature and history, to the study of classical philology ${ }^{18}$. En 1873 fut signé un accord de partenariat avec l'université de Leipzig afin de créer un séminaire philologique destiné aux étudiants russes dont plusieurs sont devenus des philologues célèbres. Le professeur de grec et de latin de Lossev au gymnasium de Novocherkassk qu'il fréquenta entre 1903 et 1911, Joseph Miksch, avait été lui-même formé au séminaire philologique de Leipzig. Lossev avoue lui-même qu'à la fin de sa scolarité au gymnasium il «était un philosophe et un philologue

16. F. Nethercott, Russia's Plato. Plato and the Platonic Tradition in Russian Education, Science and Ideology (1840-1930), Aldershot, Ashgate Publishing Ltd., 2000, p. 49.

17. Ibid., p. 24 .

18. Le but de ces réformes était lié à la lutte contre le désordre social. Le ministre de l'Éducation nationale D. A. Tolstoï (1866-1880) et son successeur I. D. Delianov (1882-1898) «saw in it an effective measure to thwart the ambitions of young 'nihilists' whose wholesale renunciation of tradition meant, among other things, rejection of classical and humanistic education" (F. Nethercott, Russia's Plato..., p. 24). Le résultat fut l'essor de la philologie classique russe dans les dernières décennies du XIX ${ }^{\mathrm{e}}$ siècle. 
classique entièrement formé ${ }^{19}$. Après le gymnasium, Lossev passe quatre années d'études, à l'université de Moscou, à la faculté historique et philologique où il s'inscrit simultanément dans la section de philosophie et dans celle de lettres classiques, et où après la soutenance de son mémoire Du sentiment du monde chez Eschyle il obtient un poste dans la section de philologie classique afin de préparer le concours au titre de professeur de lettres classiques. En résumé, il s'agit d'un philologue classique, d'un universitaire brillant, dont le parcours exemplaire n'a rien d'aberrant.

D’autre part, les références platoniciennes de Lossev sont parfaitement documentées. Il se réfère à Ueberweg, Stenzel, Ritter, Robin, Natorp, Gomperz, Zeller, Trendelenburg, Schleiermacher, Susemihl, Windelband, Stallbaum, Bonitz entre autres savants. Riche de sa culture Lossev, porte un regard critique sur les études platoniciennes en Russie. On peut bien juger de son sentiment de supériorité à partir de la remarque suivante: "La révolution qui a détruit l'ancienne école n'a pas détruit la recherche savante sur Platon pour cette simple raison qu'il n'y avait rien à détruire car les études platoniciennes n'existaient pas à Moscou ${ }^{20}$.

En revanche c'est avec le travail des philologues allemands que Lossev compare son propre commentaire:

Simultanément et parallèlement à moi, travaillaient sur Platon en Occident des hommes de l'importance de Natorp et de Cassirer; ce qui me distingue d'eux, ce n'est pas qu'ils aient eu une avance sur moi quant au travail, mais le fait d'un net décalage dans la publication de nos travaux respectifs; quant au reste, il s'agissait bien du même Platon que celui auquel je consacrais mes efforts, coincé que j'étais dans un pays sauvage et entièrement coupé, pendant les années de la révolution, des publications savantes de l'Occident ${ }^{21}$.

Ces considérations nous font donc rechercher la raison de la méthode «exégétique » retenue par Lossev ailleurs que dans son manque de culture académique et philologique. Il ne s'agit pas d’un enthousiaste débridé qui aurait confondu sa voix avec celle de Platon par manque de conscience d'une distance critique nécessaire. Tout au contraire, Lossev supprime

19. A. Takho-Godi, Lossev, Moskva, Molodaia Gvardia, 1997, p. 26.

20. А. Lossev, Ocherki..., p. 695 : «Революция, разрушившая старую школу, не разрушила науки о Платоне - по той простой причине, что и разрушать было нечего: никакого платоноведения в Москве и не существовало».

21. Ibid.: «Параллельно и одновременно со мною на Западе работали над Платоном такие большие люди, как Наторп и Кассирер; не реньше меня по самой работе, но значительно раньше по опубликованию работ, они построили такого Платона, которого строил и я, сидя в дикой стране и будучи в годы революции совершенно оторван от научной литературы Запада». 
consciemment cette distance critique, en partant de raisons qui lui sont propres. Quelles sont ces raisons?

Pour répondre à cette question il nous faut examiner la position herméneutique de Lossev, telle qu'il l'a exprimée lui-même et telle qu'elle apparaît réellement dans son travail. Pour la résumer en une phrase, on pourrait dire que, pour lui, toute compréhension plonge en fin de compte ses racines dans la sphère du préjugé. Il n'est pas gênant d'avoir des préjugés; ce qui est véritablement gênant et naï, c'est de ne pas être conscient de ses propres préjugés et de croire en la possibilité d'une interprétation objective, «scientifique» pourrait-on dire. L'interprétation objective, c'est-à-dire accessible à tout interprète, quel que soit le point de vue historique ou culturel où il se place, n'existe tout simplement pas selon Lossev. Il ne reste plus alors qu'à s'appuyer sur ses propres préjugés, pourvu qu'ils soient conscients et bien élaborés. Lossev entre ainsi en débat avec la philologie objective pour laquelle un tel point de vue est inacceptable:

Les philologues fantasment sur l'exactitude et l'objectivité. J'ignore à quel degré de précision atteignent les historiens, les linguistes, les historiens d'art, etc., dans le domaine des lettres classiques, mais je sais parfaitement que les historiens de la philosophie grecque la comprennent toujours dans les limites et à la mesure de leurs propres conceptions ${ }^{22}$.

Croire en une compréhension entièrement objective revient à appliquer à la compréhension philosophique des normes d'objectivité qui lui sont étrangères. Ce type d'objectivité, recherché notamment par la philologie, s'est constitué lui-même au sein d'une certaine tradition que l'on ne peut en aucun cas présenter comme une exigence universelle. Lossev se montre très critique à l'égard du contexte culturel européen profondément marqué par le rationalisme et par une valorisation démesurée des sciences de la nature. Ce monde mécaniste inauguré par Descartes a laissé l'empreinte d'un «schématisme mortifère» sur les études platoniciennes. C'est pourquoi, selon Lossev, "Platon, à l'aube du $\mathrm{XX}^{\mathrm{e}}$ siècle, est plus insaisissable et plus embrouillé qu'au cours du Moyen Âge ${ }^{23}$.

Lorsqu'un connaisseur de Platon aussi informé que K. Ritter considère comme légitime d'interpréter tous les cas où il [Platon] fait usage d'eidos et d'idée dans l'esprit de la logique formelle; lorsqu'un philosophe aussi honorable, à

22. Ibid., p. 139: «Филологи грезят о точности и объективности. Я не знаю насколько точны историки, языковеды, историки искусств и пр. и пр. в области классической древности. Но я прекрасно знаю, что историки древней философии всегда понимают древнюю философию в границах и в меру собственных воззрений ».

23. Ibid., p. 238 : «На заре XX в. Платон непонятнее и запутаннее, чем в Средние века». 
maints égards, que P. Natorp ne voit dans ces concepts qu'une découverte des «formes a priori de l'entendement ${ }^{24}$, il est clair que ce n'est pas parce que ces auteurs (qui ont d'ailleurs consacré toute leur vie à l'étude de Platon) manquent d'acuité critique, mais parce que cette période de l'histoire de la philosophie, dite des Temps Modernes, se distingue radicalement de la philosophie antique et ne peut que la défigurer par l'étroitesse de son rationalisme ${ }^{25}$.

La philologie objective, héritière de l'époque positiviste et adversaire résolu de Lossev, est objet de critiques violentes, parfois mal maîtrisées :

Un «philologue objectif» qui étudie, par exemple, les notions d'eidos et d'idée chez Platon, en soutenant l'indépendance de la philologie vis-à-vis de la philosophie, et tout spécialement des conceptions philosophiques du chercheur lui-même, n'est qu'une victime pitoyable de conceptions philosophiques arbitraires et fragmentaires qui lui ont été léguées par les manuels scolaires qu'il a lus lorsqu'il préparait un examen de programme universitaire. Eh bien non! puisque une culture philosophique est indispensable à l'interprétation de la terminologie platonicienne, je préfère dépendre consciemment dans mes recherches de tel ou tel système rigoureusement réfléchi plutôt que soumettre réellement, quoique inconsciemment, la philologie aux préjugés philosophiques hasardeux et décousus, au nom d'une objectivité philologique inexistante et de sa fausse indépendance à l'égard de la philosophie ${ }^{26}$.

24. Signalons que Lossev aurait pu faire une critique analogue à Joseph Moreau qui dans sa thèse de doctorat, publiée en 1939, écrivait ceci à propos de la théorie des Idées telle que Platon la présente dans le Phédon (74a-76a): «L'Idée de l'égal ou du double ne se tire pas de la comparaison des objets égaux ou doubles [...]. Une telle notion n'est donc pas abstraite, mais posée a priori; c'est une essence purement nominale, qui n'a pas à correspondre avec un cercle d'existence, une extension préalablement tracée; tirant tout son être de la décision mentale qui la pose, elle peut demeurer toujours identique à elle-même», J. Moreau, La construction de l'idéalisme platonicien, Paris, Boivin, 1939, p. 314. Le titre même, l'«idéalisme platonicien ", fera frémir un commentateur qui pense que l'intelligible existe «en soi» comme une «réalité» autonome.

25. A. Lossev, Ocherki..., p. 238: «Когда такой знаток Платона, как К. Риттер, почт все случаи употребления эйдоса и идеи находит возможным трактовать в духе формальной логики; когда такой во многих отношения почтенных философ, как П. Наторп, находит в этих понятиях только открытие “априорных форм рассудка", - то ясно, что дело не в недостатке критической остроты у Риттера и Наторпа (занимавшихся Платоном целую жизнь), а в том, что весь период философии, именуемый новоевропейским, в корне отличен от античной философии и в своей рационалистической ограниченности может ее только исказить».

26. Ibid., p. 140 : «"Объекивный филолог”, изучающий, скажем, понятия эйдоса и идеи у Платона и утверждающий, что в данном случае филология не зависит от философии, и в частности от философских воззрений самого исследователя, есть только жалкая жертва случайных и отрывочных философских воззрений, оставшихся у него от школьных учебников, которые он когда-то, проходя учебный план в университете, читал для сдачи экзамена . 
Aussi, comme il est tout à fait impossible d'éliminer toute intrusion de la part du sujet, l'interprétation qui se veut objective est-elle inconsciemment subjective et instille-t-elle à son insu des préjugés dans le commentaire savant qui en devient un commentaire en partie naï.

Nous avons délibérément employé le terme de "préjugé» par allusion à l'herméneutique gadamérienne que Lossev en 1930 ne pouvait connaître. Lossev n'emploie pas le terme "préjugé», ou bien, s'il le fait, c'est sans l'intention de lui conférer une connotation définie. Cependant le fait de voir en Lossev le précurseur de l'herméneutique gadamérienne explique rétrospectivement ce qui se pratiquait en Russie dès les années 1920, fût-ce seulement par anticipation intuitive. Bien entendu, ce parallèle avec Gadamer peut être maintenu sous réserve seulement qu'on n'oublie pas que les quelques passages dans lesquels Lossev exprime sa position herméneutique n'ont pas la même valeur qu'un livre comme Wahrheit und Methode spécialement consacré aux problèmes de l'objectivité dans les sciences humaines.

Ce qui justifie le mieux ce rapprochement avec l'herméneutique de Gadamer c'est bien davantage probablement la conscience qu'a eue Lossev du conditionnement historique de toute compréhension. «Toute compréhension est historique par nature et il ne peut en aller autrement, car l'histoire elle-même n'est autre chose que la compréhension en devenir de l'être $»^{27}$. Et on lit plus loin: «Un être qui ne saurait aucunement être compris n'est pas réel. C'est un être abstrait $»^{28}$.

Le fait qu'il n'y a pas de pensée hors de l'histoire ne veut pas dire pour autant que tous nos efforts de comprendre aient un caractère contingent.

J'estime que toutes les interprétations du platonisme qui ont existé dans l'histoire étaient également nécessaires et ne sont rien d'autre que la nécessité dialectique de l'histoire elle-même. Que telle ou telle interprétation du platonisme soit liée à telle ou telle époque ne veut pas dire qu'elle est relative et subjective. Cela signifie seulement qu'elle se donne comme la vérité pour une époque donnée ${ }^{29}$.

27. Ibid., p. 685: «Всякое понимание по природе своей исторично и иным не может и быть, ибо сама история есть не что иное, как становящееся понимание бытия ».

28. Ibid.: «Бытие, никак не понимаемое, не есть реальное бытие. Это абстрактное бытие».

29. Ibid.: «Я думаю, что все понимания платонизма, существовавшие в истории, одинаково необходимы и суть не что иное, как диалектическая необходимость самой истории. Пусть то или другое понимание платонизма связано с той или другой эпохой. Это не значит, что оно “относительно” и “субъективно”. Это значит только то, что оно является истиной для данной эпохи». 
Quelles sont les conséquences d'une telle position pour l'interprétation de Platon? "Il y a autant de figures de Platon qu'il y a eu de systèmes et d'intuitions philosophiques ${ }^{30}$, dit Lossev. Cela est aussi vrai pour sa propre interprétation de Platon, fruit de son époque historique et fondée sur des préconceptions qui en résultent. Mais plutôt que d'en subir des effets négatifs, elle en est tout au contraire enrichie, ce qui conduit Lossev à se féliciter de ses propres préjugés et à suggérer d'exploiter leur valeur herméneutique.

Notre compréhension est ainsi propre à une époque précise et à des orientations particulières et, surtout, à une intuition du monde donnée et à une vision du monde spécifique, et, ce qui est plus important encore, à une certaine attitude face au monde. Je ne le redoute pas, au contraire, je le pose ouvertement comme un principe et je mets en accusation tous ces innombrables historiens de la philosophie qui, se prononçant sur le platonisme du point de vue d'une époque historique donnée (il est impossible de faire autrement), l'ont dissimulé aux autres autant qu'à eux-mêmes en dupant tout le monde, et d'abord eux-mêmes, au nom d'une «objectivité» fausse et d'une «scientificité» illusoire ${ }^{31}$.

Après cette description de la méthode historico-philosophique de Lossev, le moment est venu de l'illustrer par quelques exemples.

Pour commencer, il serait utile de présenter la vision lossevienne de l'ensemble de dialogues platoniciens à partir des Essais sur le symbolisme et la mythologie antiques, un grand ouvrage presque entièrement consacré à Platon, et dans lequel Lossev entreprend une analyse systématique de l'ensemble des dialogues, vus sous l'angle de la «théorie platonicienne des Idées dans son développement systématique ${ }^{32}$.

Lossev établit un classement des dialogues platoniciens en cinq «stades dialectiques» qu'on a choisi par commodité de représenter sous la forme d'un tableau:

30. A. Lossev, Ocherki..., p. 685 : «Платонов столько же, сколько было философских систем и интуиций ».

31. Ibid.: «Итак, наше понимание есть понимание, специфическое для определенной эпохи и определенных настроений и, главное, для определенного мирочувствия и мировоззрения и еще важнее, для определенного мироотношения. Я этого не боюсь, а просто выставляю открыто как свой принцип и обвиняю всех тех бесчисленных историков философии, которые, произнося суждения о платонизме с точки зрения определенной исторической эпохи (иначе никакое суждение не может и осуществиться), скрывали это и от себя и от других и обманывали всех, а прежде всего самих себя, призрачной “объективностью" и “научностью”".

32. Ibid., chap. IV, p. 287-708 


\begin{tabular}{|l|l|}
\hline \multicolumn{1}{|c|}{ Nom du stade dialectique } & \multicolumn{1}{c|}{ Dialogues qu'il contient } \\
\hline Naïf et réaliste & $\begin{array}{l}\text { Théagès, Criton, Apologie de } \\
\text { Socrate }\end{array}$ \\
\hline Phénoménologique et descriptif & $\begin{array}{l}\text { Ion, Alcibiade I, Charmide, } \\
\text { Protagoras, Lachès, Eutyphron, } \\
\text { Hippias majeur, Lysias, Hippias } \\
\text { mineur, Euthydème }\end{array}$ \\
\hline Transcendantal & $\begin{array}{l}\text { Ménon, Théétète, Banquet, Gorgias, } \\
\text { Cratyle, Phédon, Phèdre }\end{array}$ \\
\hline Dialectique & $\begin{array}{l}\text { Sophiste, Parménide, Philèbe, } \\
\text { Politique, République, Timée }\end{array}$ \\
\hline Arithmologique & $\begin{array}{l}\text { L'enseignement oral de Platon et les } \\
\text { informations communiquées par } \\
\text { Aristote sur le Platon tardif }\end{array}$ \\
\hline
\end{tabular}

Un «stade dialectique» n'est ni chronologique ni strictement atemporel, mais proprement dialectique:

Selon moi, le «stade» est dialectique, c'est-à-dire qu'il présuppose une corrélation logique interne. Il est, en outre, historique au sens dialectique (et non pas chronologique), c'est-à-dire qu'il implique une irréversibilité et une orientation, très précisément une direction ascendante. En fin de compte tous les stades sont tendus vers un but unitaire qui seul leur confère le statut de $\operatorname{stades}^{33}$.

Il est inutile d'exposer la totalité des arguments de Lossev qui l'ont conduit à cette systématisation de l'œuvre platonicienne. On se contentera de dessiner le cadre schématique de cette systématique dialectique.

Première remarque à faire au sujet de ce classement : les parallèles avec la philosophie moderne y sont si fréquents qu'on ne saurait mieux illustrer le fameux aphorisme de Whitehead («La philosophie occidentale n'est qu’une

33. Ibid., p. 293: «Ступень в моем трактовании диалектична, т.е. предполагает внутреннюю логическую взаимосвязь. Кроме того, она в диалектическом (а не хронологическом) смысле исторична, т.е. предполагает неповторимость и направление, а именно восхождение. Наконец, всеми ступенями руководит единая цель, с точки зрения которой они лишь и являются ступенями». 
série de notes de bas page aux dialogues de Platon ${ }^{34}$ ) qu'en reprenant les commentaires de Lossev. D'autre part, il faut être averti du fait que Lossev n'est pas toujours soucieux de lever l'ambiguïté de termes à l'héritage aussi chargé que "transcendantal», "Eidos», "phénoménologie» et autres et qu'il est donc nécessaire de rechercher à ses risques et périls le sens exact de leur emploi chez Lossev, afin d'éviter des équivoques fâcheuses.

Le premier stade de la théorie des Idées qualifiée de "naiff et réaliste» consiste en un «constat naïf et réaliste de la réalité sensible immédiate » ${ }^{35}$. «Il s'agit là de l'être que Platon voit et entend de façon immédiate, qu'il perçoit et qu'il constate en dehors de toute réflexion. Il sera transfiguré par la suite lorsque le philosophe entendra l'élever à la sphère de son idéalité » ${ }^{6}$. Il n'y a, pour l'instant, aucune théorie des Idées mais seulement des constats naïfs, qu'il s'agisse de la réalité du démon de Socrate dans le Théagès et dans l'Apologie de Socrate, ou bien de celle de l'ordre social éternel et autonome sous forme de la personnification des lois dans le Criton. Lossev écrit:

Les questions concernant l'«inspiration » tout comme les considérations sur l'Hadès, sans parler des problèmes de l'ordre politique, Platon les abordera plus tard. Mais nulle part ces questions ne sont traitées sur le mode du réalisme naif et du vécu immédiat, à l'exception des trois dialogues qu'on vient de mentionner. L'«inspiration» du Phèdre et l'ordre social de la République, comme il s'avérera plus loin, sont soit des constructions directement dialectiques, soit relevant de telle ou telle systématisation philosophique ${ }^{37}$.

Est-ce que ces dialogues sont véritablement les premiers dialogues de Platon? Ce n'est pas de toute évidence la question qui intéresse Lossev. Sa curiosité est ailleurs: il cherche à définir le commencement de la théorie platonicienne des Idées non pas au sens chronologique du terme, mais en tant que la première attitude de la conscience face à l'être. "Sans aucun doute, c'est une attitude particulière de la conscience face à l'être et, comme telle,

34. A. N. Whitehead, Process and Reality, New York, MacMillan, 1929, p. 63; cité d'après P. Hadot, «Philosophie, exégèse et contresens», p. 3.

35. A. Lossev, Ocherki..., p. 299: «Констатирование, наивно-реалистическое утверждение непосредственно ощущаемой действительности».

36. Ibid.: «Это - то бытие, которое Платон непосредственно видит и слышит, непосредственно, вне-рефлективно ощущает и констатирует. Оно потом преобразится, когда философ захочет поднять его в свою “идеальную” сферу”.

37. Ibid.: «Вопросов “вдохновения”, равно как и рассуждений об Аиде, уже не говоря о проблеме государственного устройства, Платон касался и впоследствии. Но нигде эти вопросы не трактованы наивно-реалистически и непосредственно-жизненно, кроме указанных трех диалогов. “Вдохновение” “Федра” и социальный строй “Государства”, как мы увидим ниже, суть или прямо диалектические, или вообще те или иные философско-систематические конструкции. Там меньше всего “наивного реализма” ". 
elle est le premier stade de la "doctrine des Idées" ${ }^{38}$. L'être se présente à la conscience naïve et réaliste sous forme de "faits». "À ce niveau l'être n'est qu'un fait qu'on constate tout simplement, et dans lequel il n'y a pas plus d'“idéal" que dans n'importe quel constat d'un fait quelconque ${ }^{39}$.

Les termes de "réalisme naif » et de "fait» mis en opposition à «idée» sont à notre avis une réminiscence évidente de l'attitude naturelle de Husserl, et le fait que ce réalisme naïf est par la suite dépassé par le «stade phénoménologique» confirme d'autant plus cette impression ${ }^{40}$.

L'intention contenue dans les dialogues rattachés au stade suivant, "phénoménologique et descriptif», consiste dans le dépassement du réalisme naif du stade antérieur.

Dans les dialogues auxquels on a <alors > affaire, Platon persistera à raisonner ainsi: je tiens à définir le courage, l'amitié, la vertu, entendus, non pas dans la diversité des faits, mais selon leur essence, selon leur idée. Les actions vertueuses, par exemple, sont légion. Si on ne prête attention qu'aux actions et non à ce qui les rend vertueuses, elles cesseront d'être vertueuses; si grande que soit l'étendue de nos descriptions, nous nous enfoncerons dans une sphère qui n'a rien de commun avec la vertu en soi. Aider un malade est vertueux, tout comme de tuer un ennemi pour le salut de sa patrie. S'approprier le bien d'autrui n'est pas vertueux, sauf s'il s'agit de parents par rapport à des enfants insensés, etc., etc. Peut-on comprendre ce qu'est la vertu ou l'action vertueuse à partir de ces données contradictoires? Il est clair que non; sous un même terme vont se fourrer plusieurs concepts contradictoires. Comment se tirer d'embarras? D'une seule manière: examiner non pas des milliers d'actions vertueuses, mais la vertu elle-même, l'essence même, la forme même de la vertu, cela même que nous appelons ou convenons d'appeler vertu ${ }^{41}$.

38. Ibid, p. 299: «Это, несомненно, есть определенное отношение сознания к бытию и как таковое - первая ступень "учения об идеях" ».

39. Ibid.: «На этой ступени бытие есть пока еще просто констатируемый факт, в котором “идеального" не больше, чем во всяком ином констатировании какого бы то ни было факта».

40. Sur la présence de la phénoménologie avant la Révolution en Russie, voir l’article «Husserl en Russie» dans le Dictionnaire de la philosophie russe, Lausanne, l'Âge d'homme, 2010, p. 349-351 et l'ouvrage de M. Dennes, Husserl-Heidegger. Influence de leur cuvre en Russie, Paris, L'Harmattan, 1998. On rappellera notamment que les Recherches logiques ont été traduites en russe dès 1909 (sous la dir. de S. Frank).

41. A. Lossev, Ocherki..., p. 310-311: «В диалогах, относящихся сюда, Платон не раз будет рассуждать так: я хочу определить мужество, дружбу, добродетель - не в смысле отдельных фактов а по их сущности, по их идее. Добродетельных поступков, напр., очень много. Если обращать внимание только на самые поступки, а не на то, что делает их добродетельными, то они, конечно, перестанут быть добродетельными; и сколько бы мы их ни описывали, мы будем углубляться в сферу, ничего общего не имеющую с добродетелью как таковой. Помогать больному - добродетельно, убивать врага ради спасения отечества - добродетельно. Присваивать чужое - 
Affrontant le problème de la connaissance, Platon parvient à une opposition stricte entre l'idée, l'eidos, et les données de l'expérience. Afin de dégager l'essence du phénomène il faut s'abstenir des «faits» de l'expérience. L'idée se manifeste ainsi comme la réalité en soi, indépendante de celle des choses. Lossev écrit:

Telle est la position philosophique de Platon à ce stade. Le réalisme naïf de la phase précédente est désormais abandonné. La connaissance et l'être ne sont plus alors perçus dans leur donné immédiat, la réalité est analysée du point de vue de son aspect délibérément sélectionné et abstraitement visé, du point de vue de la connaissance, pour autant qu'est posée l'exigence de fournir l'analyse essentielle de la connaissance et de la conscience dans leur pureté, leur pleine indépendance et leur pleine autonomie ${ }^{42}$.

Mais ne confondons pas la mise hors circuit des données d'expérience avec la négation de la réalité des choses sensibles.

Il est facile de déformer cette position de Platon en l'interprétant de manière antiréaliste. Gardons-nous de le faire. De ce que le philosophe analyse la connaissance pure en tant que telle il ne résulte pas que Platon nie dès lors toute autre réalité des choses. C’est là ce que les ignorants retiennent d'ordinaire de la phénoménologie. Vous parlez de connaissance, et non pas de choses, $\mathrm{Ah}$ ah! Vous niez donc les choses. Voilà la leçon enfantine et simpliste qu'on tire d'ordinaire de la phénoménologie. Or, si la phénoménologie s' « abstient» des «faits» ${ }^{43}$, ce n'est pas pour autant qu'elle veuille les nier. Qu'on s'en «abstienne» ou non, cela n'a aucune incidence sur les faits eux-mêmes, et ce n'est pas ainsi qu'il faut s'y prendre avec les faits. Si la phénoménologie nie les faits, c'est par convention, de manière relative, provisoirement, c'est dans le seul but de faire apparaitre la vérité qui advient au-delà d'eux et qui non

недобродетельно. Присваивать чужое в том случае, когда речь идет о родителях в отношении неразумных детей, - добродетельно. И т. д., и т. д. Можно ли из этих противоречивых фактов и поступков понять, что такое добродетель и что такое даже добродетельный поступок? Явно, нельзя; под одним и тем же термином у нас будет крыться несколько взаимно-противоречащих понятий. И какой же выход? Выход единственный: рассматривать не тысячи добродетельных поступков, а саму добродетель, самую сущность добродетели, самую идею добродетели, то самое, что мы называем или условливаемся называть добродетелью ».

42. A. Lossev, Ocherki..., p. 311: «Такова философская позиция Платона на этой ступени. Тут уже покинут наивный реализм прежней ступени. Знание и бытие теперь уже не просто ощущается в своей непосредственной данности, но анализируется действительность с точки зрения произвольно выбранной намеренно-абстрактной ее стороны - именно, со стороны знания, причем выставляется требование дать существенный анализ знания и сознания в его чистоте, полной независимости и самостоятельности ».

43. Cette attitude où la pensée met entre parenthèses l'attitude naturelle est l'épokhè proprement dite (voir E. Husserl, Ideen I, $\$ 32$ ). 
seulement ne les contredit pas mais, bien au contraire, ne vise qu'à les faire accéder à la conscience et à l'intelligibilité ${ }^{44}$.

Le troisième stade, «transcendantal», aborde la question de la "réalité effective du sens dans sa fonction de rendre intelligible la réalité immédiate et sensible des données de l'expérience». Ce faisant, elle élabore, d'après Lossev, la conception du «symbole en tant que principe transcendantal».

Mettant ses pas dans ceux de Schelling, Lossev entend par symbole «l'unité indissociable du général et du particulier, de l'idéal et du réel, de l'infini et du fini» ${ }^{45}$, définition qu'il tire du $\$ 39$ de la Philosophie de l'art du philosophe allemand: "Darstellung des Absoluten mit absoluter Indifferenz des Allgemeinem und Besonderen im Besonderen ist nur symbolisch möglich» ${ }^{46}$. Lossev ne donne pas de définition de ce qu'il entend par symbolisme transcendantal. Mais on peut comprendre, à partir du contexte, qu'il s'agit de l'union indissociable, c'est-à-dire symbolique, du général et du particulier, posée comme principe transcendantal de la connaissance et de l'être.

La méthode transcendantale vise à réconcilier l'essence et le phénomène :

Cette voie et cette méthode entendent réaliser la coïncidence du phénomène et de l'essence, de la chose et de l'idée. Nous avons déjà vu que c'est le destin même de ses recherches internes qui met Platon sur la voie de cette synthèse. On ne peut pas se débattre impunément dans l'élément des sensations immédiates: elles vont nous tromper. On ne peut pas non plus réduire impunément la philosophie à une «abstention» des faits: les eidè abstraits vont nous tromper également. Tant que les faits eux-mêmes ne deviennent pas des significations, et tant que celles-ci ne deviennent pas des faits perçus naïvement et immédiatement, le philosophe demeurera condamné à la mort dans la chaleur suffocante des abstractions ${ }^{47}$.

44. A. Lossev, Ocherki..., p. 310: «Легко исказить эту позицию Платона и истолковать ее антиреалистически. Этого не следует делать. Из того, что философ анализирует чистое знание как таковое, отнюдь не вытекает того, что Платон отрицает тут всякую иную реальность вещей. Так обычно люди невежественные понимают феноменологию. Вы говорите о знании, а не о вещах: ага! значит, вы отрицаете вещи. Вот этот примитивнодетский вывод, который делают обычно из феноменологии. Конечно, вовсе не для того феноменология “воздерживается” от “фактов”, чтобы отрицать эти факты. Факты меньше всего зависят от нашего “воздержания” от них или “невоздержания”, и не так нужно бороться с фактами. Феноменология отрицает факты условно, относительно, покамест, с единственной целью изобразить только то, что поистине выходит за их пределы, хотя и не только не противоречит им, но, наоборот, всецело направлено на их осознание и осмысление».

45. Ibid., p. 20.

46. F. W. J. Schelling, Ausgewählte Schriften, Francfort, Suhrkamp, t. 2, 1985, p. 234.

47. A. Lossev, Ocherki..., p. 365-366: «Этот путь и этот метод хочет совместить явление и сущность, вещь и идею. Мы уже видели, как приводит Платона к этому совмещению 
La synthèse transcendantale s'effectue dans les conditions du maintien de l'opposition entre l'essence et le phénomène, envisagés comme deux réalités indépendantes.

La position philosophique qui se laisse apercevoir continue à s'appuyer sur le fait de la différence et de l'incompatibilité absolues entre l'idée et la réalité effective, sur le fait d'une entière opposition entre l'essence et le phénomène; tout en prenant ces deux éléments dans leur capacité à se réverbérer mutuellement dans leur corrélation signifiante ${ }^{48}$.

Dans le cadre d'une telle synthèse, l'essence est conçue comme la possibilité, la loi et la méthode des phénomènes empiriques, tandis que les phénomènes sont envisagés sous l'angle de leur structure essentielle:

Ainsi, la position philosophique envisageable, prenant l'essence et le phénomène dans leur différence absolue de fait et en même temps dans leur réverbération mutuelle signifiante, amène l'essence et le sens à devenir ce qu'ils sont appelés à être et à se transformer en un être doté d'une fonctionnalité pure et d'un dynamisme méthodique; quant à la réalité effective et aux choses, elles sont amenées à se manifester sous l'angle de leur profonde structuration intelligible et de leur agencement substantiel ${ }^{49}$.

L'insuffisance du transcendantalisme réside aux yeux de Lossev dans l'incapacité où il est d'aboutir à la synthèse ultime entre le phénomène et l'essence:

Il est totalement exclu d'accepter que cette position philosophique soit la synthèse complète et ultime de la réalité idéale et de la réalité effective, de trouver ici la solution véritable de la question relative à la corrélation du phénomène et de l'essence, que l'identification au plan de l'intelligible soit la seule identification possible. Quant à dire d'où provient l'opposition même

самая судьба его внутренних исканий. Нельзя безнаказанно барахтаться в стихии непосредственных ощущений: они обманут. Нельзя безнаказанно и сводить свою философию на “воздержание” от фактов: абстрактные эйдосы тоже обманут. Пока сами факты не станут смыслами, а смыслы - наивно и непосредственно ощущаемыми фактами, до тех пор философ остается осужденным на смерть в духоте абстракций ».

48. A. Lossev, Ocherki..., p. 367: «Итак, изображаемая философская позиция продолжает стоять на факте абсолютной раздельности и несовместимости идеи и действительности, на факте полного противостояния сущности и явления; но эта позиция вместе с тем берет эти две стихии в их взаимоотраженности и смысловой взаимосвязи ».

49. Ibid., p. 370 : « Итак, рассматриваемая позиция, беря сущность и явление в их абсолютной фактической раздельности и в тоже время в их смысловом взаимоотражении, приводит сущность и смысл к становлению сущности и смысла и к превращению в чисто функциональное и динамически-методическое бытие, а действительность и вещи - к выявлению преимущественно структурно-осмысленной и закономерно-существенной их стороны». 
de l'idée et la chose, c'est là la question à laquelle la philosophie à ce stade ne peut apporter de réponse. Et si cette réponse est introuvable, tous les efforts qu'on y déploie sont dépensés en pure perte, même s'ils sont valables en soi ${ }^{50}$.

C'est le stade «dialectique» qui apporte enfin la solution au problème de l'origine de l'opposition de l'idée et de la chose. La question centrale de la dialectique est la suivante: "comment l'être engendre-t-il son autre, son non-être? "51. Parmi les six dialogues de ce stade (le Sophiste, le Parménide, le Philèbe, le Politique, certains livres de la République, et le Timée), Lossev fait un sort particulier au Sophiste et au Parménide. Il ne se préoccupe pas de la diairesis ou de la sunagogè du Phèdre, de la question de la conformité de l'essence et du nom dans le Cratyle, et encore moins de la pratique de la discussion (dialegesthai) du Gorgias ou du Protagoras, ni en règle générale de la modalité interrogative du savoir suggérée par la forme dialoguée dans laquelle Platon a exprimé sa philosophie ${ }^{52}$.

L'accent principal est mis sur le Sophiste avec sa théorie des «grands genres» (254b-c), et sur le Parménide. Le passage du Sophiste au Parménide a pour condition la nécessité qu'éprouve Platon de réunir les megista genè dans une unité plus universelle encore: celle de l'un. "C'est pourquoi on a tour à tour l'analyse de l'un sous ses divers formes et aspects et l'analyse du rapport de l'un à l'être et aux autres catégories. C'est de quoi traite précisément le Parménide " ${ }^{53}$. La conclusion à laquelle aboutit la dialectique du Parménide consiste dans l'affirmation qu' «il faut que l'un soit non seulement un, mais aussi autre, et il faut que l'autre soit non seulement autre, mais également un ${ }^{54}$.

50. Ibid., p. 369: «Но ни в каком случае нельзя согласиться с тем, что эта философская позиция есть полнейшее и последнее синтезирование идеальной и реальной действительности, что тут мы находим подлинное решение вопроса о взаимоотношении явления и сущности, что смысловое отождествление есть вообще последнее отождествление. Откуда самая противоположность идеи и вещи - вот тот вопрос, на который не может дать ответ рассматриваемая ступень философии. А если нет этого ответа - тщетны все затраченные тут усилия, хотя, быть может, они и ценны сами по себе».

51. Ibid., p. 482: «как бытие порождает свое инобытие, свое небытие» (en italique dans le texte cité). Lossev utilise ici le terme rare «инобытие/inobytie», utilisé pour traduire les termes hégéliens Anderssein, Andersheit, Anderheit, l'altérité.

52. Voir notamment le recueil d'études La forme dialogue chez Platon, F. Cossutta, M. Narcy (dir.), Grenoble, Millon, 2001.

53. A. Lossev, Ocherki..., p. 514: «Поэтому на очереди - анализ одного, в его различных формах и видах и анализ отношения одного к сущему и прочим категориям. Этим и занимается "Парменид”".

54. Ibid.: «Одно должно быть не только одним, но в то же время и иным, а иное должно быть не только иным, но и одним ». 
Grâce à la théorie des grands genres du Sophiste surplombée par celle de l'un du Parménide, la théorie des Idées trouve son parachèvement: "L'un qui est: tel est l'organisme dialectique vivant de l'eidos susceptible de se dissocier en de nécessaires antinomies ${ }^{55}$.

Le fait que les dialogues tels que le Sophiste, le Parménide, le Philèbe, la République ou le Politique soient rattachés à l'échelle dialectique ne saurait surprendre. Il en va tout autrement du Timée qui ne mentionne même pas le verbe dialegesthai. Pourquoi est-il présent dans cette section?

L'explication est la suivante: «Le sens et l'eidos doivent prendre corps. C'est seulement alors qu'on parviendra à la sphère de la réalité effective véritable ${ }^{56}$. Toute idée doit se réaliser, devenir effective : "L'eidos doit revêtir un corps, devenir chose, se transformer en événement. L'eidos qui exprime l'intelligence sophianique doit devenir le corps de tout ce qui est, la chose universelle de l'être, l'événement englobant de la réalité effective ${ }^{57}$. Cet eidos réalisé reçoit désormais la dénomination de "mythe»: «Le mythe est l'intelligence réalisée, l'eidos donné dans la nature, l'idée affirmée concrètement ${ }^{58}$. Ainsi le Timée, ce dialogue sans véritable dialogue, ce «mythe vraisemblable» selon les termes de Platon ${ }^{59}$ "se révèle comme le point d'orgue de la dialectique platonicienne en général ${ }^{60}$.

Il n'est point difficile d'énumérer les reproches qu'on pourrait adresser à une telle interprétation de Platon. Premièrement, elle confond la lecture de Platon avec des problèmes philosophiques qui dépassent largement le cadre de la philosophie platonicienne. D'autre part, ce n'est pas une lecture impartiale: l'interprétation est étroitement associée à l'évaluation, d'habitude positive mais parfois aussi négative, des dialogues platoniciens. Platon joue souvent le rôle de porte-parole de Lossev qui s'identifie à son allié philosophique. Par exemple, au sujet des arguments du Théétète, Lossev s'exclame:

55. A. Lossev, Ocherki..., p. 514: «Одно сущее - живой диалектический организм эйдоса, расчленяемый на необходимые антиномии».

56. Ibid., p. 571: «Смысл и эйдос должны стать телом. Только тогда мы перейдем в сферу подлинно действительности».

57. Ibid., p. 572: «Эйдос должен перейти в тело, тать вещью, превратиться в событие. Интеллигентно-софийно-выразительный эйдос должен стать телом всего сущего, универсальной вещью бытия, всеобщим событием действительности. Это значит, что он должен стать космосом».

58. Ibid.: «Миф есть осуществленная интеллигенция, в природе данный эйдос, вещественно утвержденная идея ».

59. Platon, Timée, 29d: «si, en ces matières, on nous propose un mythe vraisemblable, il ne sied pas de chercher plus loin» (trad. par L. Brisson).

60. A. Lossev, Ocherki..., p. 572 : «Так Тимей оказывается венцом платоновской диалектики вообще». 
"C'est là la seule objection valable contre le sensualisme théorique " ${ }^{61}$. Ou bien encore: «Il [Platon] a montré qu'on ne peut pas penser autrement. Là réside non seulement sa dialectique mais aussi son seul mérite philosophique ${ }^{62}$. Lossev refuse le principe de l'intentio auctoris. En effet, pour lui, à l'enjeu de l'investigatio mentis auctoris se substitue la recherche de la vérité elle-même supposée révélée dans un texte philosophique. L'histoire de la philosophie tout entière est pensée sur le mode de la révélation: «S'il y a une raison à l'histoire de la philosophie, c'est parce que l'homme est incapable d'étreindre d'un coup la vérité; il ne la saisit que par fragments discontinus. L'homme meurt à la seconde même où il est saisi par l'instance ultime de la sphère de la vérité qui lui a été assignée. Il en va de même pour les philosophes ${ }^{63}$.

Lorsque Lossev analyse, par exemple, la théorie des Idées platonicienne en la transposant en termes hégéliens, husserliens, néokantiens ou autres, il mélange des concepts provenant de divers domaines et il introduit des termes et des conceptualités étrangères au texte authentique. Ce qui est vrai également pour l'usage qu'il fait en parlant de Platon de la notion de «Sophia» et de l'épithète "sophianique» dans un sens qui ne s'explique que par l'héritage théologique semi-hérétique de la philosophie russe ${ }^{64}$.

Pour parvenir à un corps de doctrine cohérent, Lossev effectue une sélection rigoureuse des éléments considérés comme valables, au prix d'un rejet de beaucoup de données considérées par lui comme "arbitraires». Le texte platonicien est habituellement réduit à son «idée principale», à son "contenu philosophique pur», et la forme dialogique est abandonnée car elle ne fait qu'obscurcir le contenu philosophique des œuvres. Il en va de même des aveux socratiques d'ignorance, Lossev prenant le soin de reformuler le raisonnement hypothétique de Socrate sur un mode catégorique, et d'apporter des réponses là où il n'y avait que des questions. Quant au classement des dialogues platoniciens effectué par Lossev, on peut se demander quel est son rapport avec Platon. N'étant ni chronologique,

61. Ibid., p. 387: «Только так и можно возразить теоретическому сенсуализму ».

62. Ibid., p. 237 : «Он показал, что и мыслить иначе нельзя. В этом не только его диалектика, но и единственная философская заслуга».

63. Ibid., p. 370 : «Потому и существует история философии, что люди не могут обнять истину сразу, а схватывают ее по отдельным кускам. И человек умирает в ту секунду, когда им охвачен последний момент в той сфере истины, которая ему суждена. Таковы и философы».

64. Là encore voir le Dictionnaire de la philosophie russe, article "sophiologie», p. 811-818; la dimension «sophianique» du monde est particulièrement méditée par Soloviov et Florenski. Pour le dire de façon très schématique, c'est la puissance par laquelle une partie du Christ, sa sagesse ou Sophia, permet la création d'un monde imparfait. 
ni simplement thématique, comment peut-il prétendre à une objectivité quelconque? Il est difficile de pardonner l'arrogance de cet interprète qui accommode le texte à sa guise!

On sera peut-être moins intransigeant au sujet de Lossev si l'on se remémore qu'il y a eu une époque dans l'histoire de la philosophie occidentale où ces fautes n'auraient pas été une exception à la règle générale. Pierre Hadot remarque très justement qu'en Occident «pendant près de deux mille ans, du milieu du IV $\mathrm{IV}^{\mathrm{e}}$ siècle av. J.-C. jusqu'à la fin du XVI ${ }^{\mathrm{e}}$ siècle, la philosophie a été conçue avant tout comme une exégèse, se rapportant à un petit nombre de textes émanant d'“autorités" dont les principales furent notamment Platon et Aristote ${ }^{65}$. Dans ce contexte, les problèmes philosophiques se posaient en termes exégétiques: "Dans la mesure où la philosophie a été conçue comme une exégèse, la recherche de la vérité s'est confondue, pendant toute cette période, avec la recherche du sens des textes “authentiques", des textes faisant autorité ${ }^{66}$. Chercher la vérité revenait à bien expliciter le texte sacré dans lequel elle était «donnée». Mais puisque l'intérêt premier était porté vers la science elle-même, les écrits du maître n'étant que son véhicule, l'activité de l'interprète visait à faire correspondre le texte avec ce qu'on considérait comme étant la vérité de la doctrine. Il en résultait, note Pierre Hadot, de multiples artifices exégétiques et souvent le texte source fut pris à contresens. C'est sous forme de ces contresens que s'opéraient l'évolution et l'innovation doctrinale en philosophie à l'ère "exégétique».

$\mathrm{Au}$ fond ce qu'on reprocherait à Lossev, interprète de Platon, ce sont les erreurs mêmes qu'on rencontre chez les commentateurs de l'Antiquité tardive que Pierre Hadot résume ainsi:

On peut énumérer brièvement les formes que peuvent prendre ces contresens et ces déformations. Tout d'abord, les exégètes systématisent arbitrairement: ils rapprochent les unes des autres des formules de l'auteur, éloignées dans leur contexte et prises formellement, pour réduire l'ensemble des textes qu'ils veulent expliquer à un corps de doctrine cohérent. C'est ainsi que l'on a pu tirer des différents dialogues de Platon une hiérarchie des êtres à quatre ou cinq étages. Ce n'est pas encore le plus grave. Consciemment ou non, la systématisation parvient à amalgamer des notions disparates qui proviennent de doctrines différentes, voire contradictoires. C'est ainsi que nous voyons les commentateurs d'Aristote donner à des textes aristotéliciens une exégèse qui utilise des notions stoïciennes et platoniciennes. D'autre part, il arrive assez souvent, notamment lorsqu'il s'agit de l'exégèse de textes traduits, que le commentateur cherche à expliquer des notions qui n'existent absolument

65. P. Hadot, «Philosophie, exégèse et contresens », p. 3.

66. Ibid., p. 6. 
pas dans l'original. [...] Sans aller toujours jusque-là, il arrive souvent que des exégèses construisent tout un édifice d'interprétations sur une formule banale ou prise à contresens. Il semble bien que toute l'exégèse néoplatonicienne $\mathrm{du}$ Parménide soit un exemple d'un tel phénomène ${ }^{67}$.

Lié, selon Pierre Hadot, au fait de l'existence d'écoles philosophiques dans lesquelles étaient « religieusement conservés la pensée, le style de vie, les écrits d'un maître ", ce phénomène a eu une longévité surprenante, depuis les présocratiques jusqu'à la fin de l'époque anté-cartésienne. L'expression la plus pure de cette tradition est l'activité des commentateurs de Platon jusqu'à la fermeture de l'école d'Athènes en 529. Elle continua ensuite grâce aux efforts de leurs successeurs dans le monde arabe et dans l'Occident latin jusqu'à la Renaissance (Marsile Ficin). À la fin de l'Antiquité on voit apparaître, à côté de l'autorité de Platon et d'Aristote, celle des Révélations : la Bible pour les juifs et les chrétiens et les Oracles chaldaïques pour les philosophes païens.

Bien entendu, il est difficile de voir une correspondance sociologique quelconque entre l'école d'Athènes et la philosophie en Russie bolchevique des années 1920. Comment alors peut-on expliquer ce fait que Lossev fait renaître la philosophie «exégétique » avec les « contresens » qui caractérisent cette forme de pensée?

Il semblerait, contrairement à sa limitation par Pierre Hadot à l'époque anté-cartésienne, que cette forme de pensée ne se soit jamais définitivement éteinte. On n'ira pas jusqu'à étudier les avatars modernes de la fonction sociale d'interprétation des textes faisant autorité, on dira seulement que l'activité d'interprétation revêt souvent chez divers auteurs les traits de l'exégèse, telle que celle-ci s'est constituée dans l'Antiquité tardive. Hegel, par exemple, définit la dialectique de Platon avec la même insouciance du texte original: «L'universel en soi - voilà ce que la philosophie platonicienne détermine de façon plus précise. Cela se présente sous plusieurs formes [...]. La forme la plus élevée que l'on trouve chez Platon est l'identité de l'être et du non-être ${ }^{68}$. Pourquoi Hegel fait-il l'éloge du Parménide en disant que "la dialectique proprement dite sous sa forme achevée est contenue dans le Parménide, le chef-d'œuvre le plus célèbre de la dialectique platonicienne ${ }^{69}$ ? Ou encore qu'est-ce qui conduit Hegel, sinon sa propre philosophie, à la division de dialogues platoniciens en «dialectique», "philosophie de la nature», et «philosophie de l'esprit»?

67. Ibid., p. 8.

68. G. W. F. Hegel, Leçons sur l'histoire de la philosophie, Paris, Vrin, t. III, 1972 (trad. par P. Garniron), p. 448.

69. Ibid. 
Une autre question, est celle de l'établissement de la chronologie des dialogues platoniciens. Ses bouleversements permanents ne prouvent qu'une chose: "It is no exaggeration to state, in general, that most pronouncements on Plato chronology were not as well-founded as they may appear to be, viewed superficially. And this is also one of the reasons for the persistent discrepancies between the chronologies suggested ${ }^{70}$.

L'interprétation personnelle, le choix en faveur de telle ou telle méthode, ou bien tout simplement la mode établie dans tel ou tel milieu académique tiennent lieu d'arguments en faveur d'une chronologie déterminée des dialogues platoniciens dans des conditions où l'on ne possède pas suffisamment d'informations à ce sujet. Dans cette situation, le commentateur a le choix entre deux solutions: soit prendre parti à ses risques et périls, soit s'abstenir de toute prise de position. Le scepticisme semble être la seule alternative à l'interprétation partiale. "The ever-growing uncertainty concerning fixed points, trends of change or development, and methods to be employed, is something explicitly admitted. More often it is to be seen in a reluctance to take a definite stand on questions of chronology» ${ }^{71}$.

Il nous semble donc qu'en reconnaissant dans l'œuvre de Lossev un prolongement vivant de la tradition néoplatonicienne (tradition qu'il a étudiée avec un enthousiasme acharné et qu'il a considérée comme la plus proche de sa propre pensée), nous nous munissons d'une bonne clé de lecture nous permettant de faire ressortir un grand nombre d'aspects de la vaste palette de cette pensée philosophique. C'est en même temps mettre en valeur le rôle tout à fait capital joué par cet auteur aussi bien pour la réception que pour l'interprétation du néoplatonisme en Russie, à l'époque où le matérialisme et le communisme ne laissaient pas beaucoup de chances à la survie de cette tradition philosophique.

Olga Ковеnкo

ENS Ulm

70. H. Thesleff, Studies in Platonic Chronology, Commentationes Humanarum Litterarum, Helsinki, Societas Scientiarum Fennica, 1982, p. 19.

71. Ibid. 